\title{
QUE INICIAL ÁTONO COMO MARCA DE MODALIDAD
}

\author{
Salvador Pons Bordería \\ Universitat de València. Grupo Val.Es.Co \\ salvador.pons@uv.es
}

\section{Resumen}

Unstressed que-inital constructions have been traditionally treated as instances of conjunction-headed structures, preceded or not by an implicit performative verb. In this paper it is argued that such an explanation runs into problems when que-constructions are faced with their interrogative counterparts, which also show unstressed-initial que.

As an alternative, que is considered a modality marker, whose main function is to show, in these constructions, the speaker's stance with regard of the proposition. In this light, a unified account of interrogative, affirmative and exclamative instances of unstressed que-initial sentences can be provided.

\section{Introducción}

No es infrecuente, ante construcciones como la siguiente:

(1). A: Que vengas

explicar la función del que inicial en función de un verbo de habla, normalmente decir, elíptico o sobreentendido, pero recuperable en el análisis, de modo que la estructura de dicho enunciado no sería (2):

(2). [o Que vengas "]

sino (3):

(3). Loigo [o que vengas $\left.{ }_{0}\right]$ "J.

Asimismo, en estructuras como (4):

(4). Que sí

existiría un verbo de decir que dominaría el resto de la estructura:

(5). Digo [que si $\left.{ }_{4}\right]$ 
Esta explicación se suele aceptar sin mayores objeciones, a pesar de que la teoría que la sustentaba, la hipótesis performativa de la semántica generativa, ya hace tiempo que demostró su inviabilidad (para una explicación en profundidad del "auge y caída de la hipótesis performativa", vid. Levinson, 1983: 236-251) y también se puede encontrar en la tradición gramatical:

En oraciones independientes que expresen deseo, el verbo va en subjuntivo [...] Su carácter desiderativo está sobradamente claro para que no necesiten estas oraciones de un verbo principal que lo exprese. A veces, sin embargo, adopta la forma de la subordinada por medio de la conjunción que. Ej.: que se alivie; que se divierta; ique entre!; ;que pase! (Gili Gaya, 1983: §115. El subrayado es nuestro).

Lo que nos proponemos en este artículo es 1) demostrar que una explicación en términos de un verbo de habla sobreentendido plantea problemas irresolubles; 2) reformular el problema a partir de los valores de que inicial átono y 3) proponer una explicación modal de dichos casos.

\section{Problemas de un verbo sobrentendido}

Obsérvese, en primer lugar, que la explicación basada en un verbo de decir sobrentendido solo se utiliza en pocos casos: por lo general, en aquellos en los que existe un enunciado encabezado por una conjunción, especialmente si esta es que. Sin embargo, si lo que estuviera poniendo de relieve esta estructura fuera la presencia de una fuerza ilocutiva determinada, dicho verbo sobrentendido debería aparecer en el análisis sintáctico de, al menos, todas las oraciones declarativas, porque la fuerza ilocutiva es una propiedad pragmática de los enunciados y, por tanto, es independiente de su estructura sintáctica, de modo que el análisis sintáctico de (6):

\section{(6). Juan come patatas}

debería de ser (7):

\section{(7). Digo que [Juan come patatas]}

pero no parece haber muchos lingüistas deseosos de suscribir esta explicación por las mismas razones que, desde los años setenta, no se acepta que la proyección superior de una oración sea un verbo performativo sobreentendido. Y si no se acepta esta relación para el caso de las oraciones declarativas sin que inicial, ¿por qué se habría de aceptar en oraciones igualmente declarativas, esto es, idénticas desde el punto de vista de su fuerza ilocutiva, solo que encabezadas por una conjunción?

Por otra parte, añadir un verbo a las oraciones con que inicial plantea problemas sintácticos propios: los enunciados con que inicial pueden incorporar un verbo de decir a su contenido proposicional, como en (8): 
Aquí, el verbo que antes estaba implícito es ahora explícito; así que, en este caso, no haría falta reponer nada. Pero esta estructura puede estar encabezada, también, por un que, como en (9):

(9). Que digo que sí

En este caso, la solución de hacer depender toda la estructura de un verbo subyacente, a diferencia de (3), crea un enunciado pragmáticamente inadecuado, si no abiertamente agramatical:

\section{(10). \#Digo [que digo que si]}

Por último, las oraciones con que inicial átono presentan alternancia entre indicativo y subjuntivo, lo que da lugar a alternancias en los verbos que encabezarían cada oración:

$\begin{array}{ll}\text { (11) a. } & \text { Que llueve/llueva! } \\ \text { b. Digo que llueve } \\ \text { c. Deseo que llueva } \\ \text { (12) } \\ \text { b. Due viene/venga! } \\ \text { c. Digo que viene } \\ \end{array}$

Y plantea el problema clásico de la determinación de la fuerza ilocutiva, sobre todo en (12.c).

Así que, para suponer que existe un verbo de habla subyacente es necesario reponer un verbo, como en (3), o un verbo más su conjunción completiva, como en (7), o nada, como en (8). Este verbo, además, podría estar implícito o explícito, según los casos, o no estar, como en (9); cambiar su naturaleza en función de la fuerza ilocutiva (11) o quedar indeterminado en ausencia de factores contextuales que aclaren la ambigüedad (12). No parece esta una explicación muy satisfactoria al problema que nos ocupa.

\section{Que como centro del problema}

La razón de esta irregularidad radica en que el diagnóstico del problema es incorrecto: el problema no está en el verbo, ya sea implícito o explícito, ni en las relaciones entre la fuerza ilocutiva y la estructura sintáctica del enunciado, sino en la conjunción. El problema es el que inicial.

La Gramática afirma que una conjunción une. La union es una función de dos lugares y comprende, por tanto, dos elementos; uno que va delante y uno que va detrás. En los casos en los que la conjunción aparece en posición inicial $-\mathrm{y}$, por ello, no tiene ningún constituyente a su izquierda que unir con el que está a su derecha-, el lingüista se ve ante un problema, que se puede resolver presuponiendo la existencia de un constituyente implícito. Esta solución no es nueva: el estudio de los marcadores ha aportado multitud de ejemplos en los que se establece una unión entre el enunciado y la enunciación, o incluso entre el enunciado y alguna proposición implícita en la memoria discursiva (Berrendonner, 1983). En el caso que nos ocupa, el verbo de habla subyacente sería el elemento repuesto, con lo 
que la conjunción ve restablecida su función original, ser un conector de dos lugares, aunque esto suponga la incorrecta opción de identificar la fuerza ilocutiva con una marca lingüística y forzar la interpretación sintáctica de la oración.

\subsection{Que inicial átono como conector}

Es posible una interpretación más adecuada de este tipo de estructuras distinguiendo los aspectos semanticos y pragmaticos que se dan en este tipo de construcciones. Garrido Medina (1998), siguiendo los presupuestos de la Gramática Funcional de Simon C. Dik, distingue dos aspectos en el tratamiento de que: uno de naturaleza sintáctica y otro de naturaleza pragmática. El primero tiene que ver con el carácter focal de las construcciones encabezadas por que átono y por la relación entre que y subjuntivo. Obsérvese que la construccion (13. a) contrasta con la mas neutra (13.b) tanto en lo referente a la modalidad oracional como en la presencia de un sujeto posverbal ( $\#_{i} Q u e$ Juan viene!). Mediante este contraste, "the focus construction represents the connection of the event to other facts represented in the discourse or in the context" (Garrido Medina, 1998).

\section{(13). a. ¡Que viene Juan! \\ b. Juan viene}

Por otra parte, en ciertas ocasiones, las oraciones introducidas por que inicial átono exigen el subjuntivo. El subjuntivo aparece con poca frecuencia en oraciones independientes y, cuando lo hace, suele estar vinculado a marcas formales (ojalá, si en oraciones independientes -Schwenter, 1999-, que) y a entornos muy restringidos (p.ej., los verbos vivir y morir: que viva, que muera-Garrido Medina, 1999: 3908-). Se trata del llamado tradicionalmente subjuntivo optativo, mediante el cual se expresa deseo o mandato.

Tanto el carácter focal como la selección del subjuntivo indican que las construcciones estudiadas tienen una vertiente gramatical. En este tipo de estructuras, que no funcionaría como conjunción subordinante ni como relativo, sino que se trataría de un conector discursivo que une enunciados independientes y al que no se le puede asignar, en ausencia de más información, un valor gramatical determinado, de modo que este se determinaria contextualmente. Estaríamos, pues, en el límite entre la gramática y el discurso. A idénticas construcciones llega Porroche (2000), quien considera que como marcador discursivo cuya función es la de introducir un comentario a propósito de otro enunciado.

El segundo aspecto es de naturaleza pragmática y se relaciona con la expresión de la sorpresa. La sorpresa se define como contraste entre la información introducida por que y la información existente en la memoria discursiva del hablante. El español codificaría en su gramática la expresión de la sorpresa mediante la estructura encabezada por que con entonación contrastiva (Garrido Medina, 1998).

En resumen, para Garrido Medina, el que inicial átono se puede explicar como un conector, que manifiesta propiedades tanto gramaticales (relación con el subjuntivo en oraciones subordinadas) como pragmáticas (expresión de la sorpresa) y cuyo estudio debe respetar ambos niveles.

Esta explicación permite delimitar la naturaleza del problema, que se hallaría, como hemos apuntado, en el que, y considerar que estas estructuras, aunque gramaticales (mejor, insertables dentro de la gramática) no se circunscriben a los usos conjuntivos clásicos, lo 
que plantea el problema de decidir si estas construcciones pertenecen al ambito gramatical, lo que supondría ampliar la gramática para dar cabida a estos ejemplos, o si su explicación tiene que ser pragmatica. Garrido Medina sugiere un análisis en dos niveles, en el que la gramática explicaría los usos de que como conector discursivo y la pragmática se ocuparía de su naturaleza enfática (Garrido Medina, 1998 48). Nótese que esta explicación es preferentemente gramatical, puesto que los usos pragmáticos se reducen a los casos de énfasis. Sin negar la vertiente gramatical de los usos de que, especialmente porque permite relacionar los valores enfáticos con la posición de foco, o enlazar los ejemplos objeto de este trabajo con los casos de que inespecífico (del tipo no me pises, que llevo chanclas), vamos a centrar nuestra explicación en la otra vertiente, en la que "que, como introductor de construcciones independientes, indica básicamente la presencia del hablante en la enunciación" (Porroche, 2000 ); es decir, en la vertiente modal. Pero para ello, será necesario tener en cuenta los valores de que inicial átono en oraciones interrogativas.

\subsection{Que inicial átono en interrogativas}

Hasta ahora hemos visto los casos de que inicial átono con modalidad oracional afirmativa y exclamativa. Existe un segundo conjunto de casos en los que que átono aparece en posición inicial, esta vez en enunciados interrogativos, tanto directos como indirectos. Reproducimos la casuística y los ejemplos que ofrecen tanto Escandell Vidal (1999: 3979 y ss.), para las interrogativas directas, como Súñer (1999: 2160 y sigs.) para las interrogativas indirectas:

1. Enunciados interrogativos directos:

(19) A: Alli probé el smorgåsbord

$B ;$ ¿Que probaste el qué? (interrogativas atribuidas de eco)

(20) A: ¿Quién te lo ha dicho?

B: ¿Qué?

A: ¿Que quién te lo ha dicho? (interrogativas atribuidas de copia)

(21) ¿Que qué hago yo aqui ahora? Déjame que te lo explique (interrogativas anticipativas)

(22) ¿Que por qué este? Como deciamos... (Escandell Vidal, 1999: 3983-4003)

2. Interrogativas indirectas

Maria me preguntó que qué era eso

Añadimos a estos el siguiente ejemplo, que se atribuye a un uso regional de que, producido por contacto con el catalán:

3. Supuesto uso regional de que

$$
\text { ¿que has llegado ya? }
$$

1 Puede que este uso se pueda explicar por causas internas, como muestra la siguiente cita de Bello: Al anunciativo que suelen acompañar otras varias elipsis que hacen muy expresiva la frase: <En fin, señora, ¿que tu eres la hermosa Dorotea, la hija unica del rico Clenardo! (Cervantes): con que tu eres. ¿que te faltan las alforjas, Sancho?> (Cervantes): con que te faltan. (Bello, 1847: 1988, \$995). 
Tanto Escandell como Súñer analizan que, en los ejemplos anteriores, como partícula citativa mediante la que se indica que la proposición encabezada por que no representa un pensamiento del propio hablante, sino un fragmento atribuible a otra persona ${ }^{2}$. Así, mediante (21) el hablante anticipa la posible pregunta que se están haciendo otros hablantes para responderla antes de que se formule; o en (23), introduce la proposición citada, actuando como frontera entre esta y la estructura introductoria. En ambos casos, que posee carácter extraoracional. En el caso de las interrogativas directas, el que átono puede preceder al pronombre interrogativo en función de sujeto (vid. ej. 21) dentro de su misma curva melódica, algo que está permitido a muy pocos elementos, entre los que se encuentra la conjunción $y$ :

$$
\begin{aligned}
& \text { a. ¿Y qué hago yo aqui ahora? } \\
& \text { b. ¿Qué qué hago yo aqui ahora? }
\end{aligned}
$$

El carácter proposicional del fragmento introducido por que se comprueba, en las interrogativas indirectas, al aplicar la prueba propuesta por Súñer (1999: 2160): cuando una estructura del tipo <que + prop> en estructuras interrogativas indirectas introduce una verdadera pregunta, esta debe estar seguida de si. En caso de que esto no sea posible, nos encontraremos no ante una pregunta indirecta, sino ante una proposición. Como se puede comprobar, la segunda opción es la correcta en los casos que nos ocupan:

En ambos casos, y como resultado de su diferente función, que átono y qué tónico pueden coocurrir, teniendo el primero ámbito sobre el segundo.

La interpretación citativa reconduce los usos de que al ámbito de los evidenciales (Dendale y Tasmowski, 2001: 343); en concreto, a los usos hearsay, por los que un hablante ofrece una información a la que ha accedido por vía indirecta.

El ejemplo (24), en el que supuestamente se refleja un uso regional del español hablado en la Comunidad Valenciana, pero que se puede atestiguar también en la cita de Bello reproducida en la nota 1 , sin embargo, no se ajusta a la interpretación citativa que acabamos de reseñar, ya que en estos casos no se repite un pensamiento atribuible a otro enunciador o punto de vista, puesto que hay una identidad entre la fuente del enunciado y el enunciador que la sustenta. Para los usos regionales, los hablantes consultados han parafraseado este tipo de enunciados con etiquetas como "sorpresa", o han asociado su uso a "situaciones inesperadas". Lo que esto sugiere es que los usos de que como partícula citativa en construcciones interrogativas se insertan en un marco más general, del que los valores citativos son subfunciones.

2 En realidad, sería más exacto hablar de "puntos de vista" (Rossari, 1994), para evitar la tentación de equiparar la fuente del enunciado con un hablante real. Así lo afirma Escandell Vidal (1999: 3967):

Basta, por tanto, con que el emisor presente el contenido de su enunciado 'como si fucra' un enunciado de otro [...] para que la presencia de que se encuentre justificada [...] Se consigue una caracerización más precisa de la contribución de que a la interpretación al afirmar que es una marca de atribución a otro del contenido transmitido.

3 Otros elementos, como pues, claro o bueno, que aparecen a la izquierda de que, no se integran en la estructura entonativa de la interrogativa, de modo que forman una unidad diferente, de rango variable, a la constituida por esta. 
Como cierre de esta sección, vamos a suscribir la interpretación citativa y vamos a plantear una explicación coherente con la anterior para los usos de que inicial átono en oraciones declarativas. De este modo, todos los empleos en los que este elemento aparece en posición inicial tendrían una explicación unitaria.

\section{Que inicial átono como marca de modalidad}

La interpretación de los usos de que en oraciones declarativas/exclamativas, por un lado, y en oraciones interrogativas, por otro, muestra una asimetría evidente, porque, mientras en el primer caso se presupone la función unitiva de que (no ya en el ámbito gramatical, sino en el pragmático), la función en el segundo es algo completamente distinto. La línea argumentativa de esta sección va en la línea de la interpretación no unitiva.

Obsérvese que, hasta este momento, se ha aceptado una idea implícita: la función de que consiste en unir, porque este es el valor que se le atribuye en la mayoría de sus apariciones. Mantener la idea unitiva obliga a pagar un peaje teórico: es necesario presuponer un elemento a su izquierda, que se debería recuperar de la memoria discursiva ¿Pero y si no tuviera dicha función? Hay una buena razón para ello, ya que no existe ningún constituyente identificable. Al liberar que de su función unitiva ya no resulta necesario recuperar ningún elemento implícito.

No es algo nuevo el que una conjunción realice funciones distintas a las de unión, y así se ha señalado en nuestra tradición gramatical, donde existen referencias a la función enfática que realizan distintas conjunciones (Salvá, 1835: 704 para pues; GRAE, 1854: 128 para $y$; Lenz, 1920: 85, para distintos elementos). El paso incorrecto, a nuestro juicio, consiste en incluir dichas funciones como variantes más o menos marcadas de la union. Sorpresa, aviso o enfado no tienen que ver con la relación entre dos constituyentes (aunque es frecuentemente la relación semántica entre los elementos de ambos constituyentes la que la provoca), sino con la actitud del emisor del mensaje hacia el mensaje transmitido, es decir, tienen que ver con una categoría pragmática diferente, a la que vamos a denominar, siguiendo la tradición alemana, y propuesta para el español por autores como Acosta (1984), Martín Zorraquino (1991), Pons Bordería (2000) o Ferrer Mora (2002) modalidad.

Para realizar esta reinterpretación, vamos a examinar a continuación los contextos de aparición de que inicial átono en enunciados declarativos y exclamativos ${ }^{4}$ :

(26) Que si (réplicas enfáticas)

(27) Que vengas (órdenes)

(28) Que te aproveche, Que viva, etc. (deseos y maldiciones)

(29) Que gaste ese dinero en tonterias!

(30) Que me ha dicho el jefe que te llame (introductor de un enunciado con estilo indirecto)

(31) Que [digo yo que] podemos comer ya (usos matizados o atenuados)

(32) Que de noche lo mataron/ al caballero (poesia popular)

(33) Que lo tuyo lo he intentado localizar (introductor de cambios de tópico)

4 Esta agrupación se debe a que muchos de estos ejemplos pueden aparecer como afirmativos o exclamativos, en función de la entonación. Así sucede, por ejemplo, con (12) o (13). 
La tipología de estos casos es diferente. En primer lugar, en los casos de (26) y (27) se encuentra un que inicial de enunciado pero no inicial absoluto, puesto que presupone un contexto previo. En ausencia del mismo, su uso resulta pragmáticamente inadecuado:
(26) A: No voy a ir
B: Si vas a ir
A: ¡Que no!
B; iQue si!
(26") A: No voy a ir
\#B; iQue si!
(27') A: Ven
$B:$ (no responde) / $B$ : No voy
$A$ : ¿Que vengas!
(27") \#A: 'Que vengas! (formulación inicial de una orden)

Lo que permite su aparición es, a nuestro entender, un contexto de insistencia, en el que se repite por segunda vez, en todo o en parte, la formulación del mensaje precedente ${ }^{5}$. Tanto ¿Que no! como iQue si! producen un resultado pragmáticamente inadecuado en (26”), porque carecen del contexto previo que justifica su aparición. El contexto no debe ser necesariamente lingüístico, puesto que puede apoyarse en la situación precedente (por ejemplo, en un hablante haciendo a otro gestos de que venga y negarse este con la cabeza). En definitiva, lo que necesita este que para aparecer no es una respuesta, sino una réplica.

Esta diferencia entre responder y replicar se marca en lenguas como el francés o el alemán mediante lexemas específicos, que forman parejas de enunciados alternantes:

$\begin{array}{ll}\text { (34) } & \text {-Oui! } \\ & - \text { Non! } \\ & - \text { Mais si! } \\ & - \text { Mais non! } \\ \text { (35) } & -J a ! \\ & - \text { Nein! } \\ & - \text { Doch! } \\ & - \text { Nein! }\end{array}$

El español, por el contrario, no habilita un lexema específico, sino que recurre a un procedimiento sintáctico, es decir, a una construcción encabezada por un signo específico -que- y acompañada de una curva entonativa marcada.

Las construcciones de (28) y (29) se caracterizan no solo por la presencia de que, sino por ser oraciones independientes construidas con subjuntivo (el tradicional subjuntivo optativo). Los casos de subjuntivo con oraciones independientes (vid. una descripción en Garrido Medina, 1999: 3908-3909) son relativamente infrecuentes y se caracterizan por estar precedidos, en la mayor parte de sus apariciones, por una marca específica: ojalá, si, asi, que. La presencia del subjuntivo y la de que nos parecen manifestaciones de un mismo 
fenómeno: la actitud del hablante ante lo enunciado; son marcas de que la oración no se debe evaluar por su factualidad, sino por el grado de adhesión del hablante hacia su contenido.

Por el contrario, considerar que como marcador discursivo obliga a recuperar una o varias proposiciones implícitas, a la(s) que se uniría el enunciado encabezado por que. Esto supone que, si dichos implicitos faltan, estos enunciados serían difícilmente procesables (tanto como una oracion coordinada a la que le faltara el primer miembro); por el contrario, si lo que falta es el conector, el enunciado restante sería totalmente gramatical (tanto como el primer miembro de una coordinación oracional). Sin embargo, la supresión del que inicial átono ofrece diferentes resultados en funcion del tipo de enunciado en que se inserte: gramaticalidad en (36); un enunciado pragmáticamente inadecuado en (37) y abiertamente agramatical en (38), (39) y (40):

(36) iQue viva España! iViva España!

(37) iQue viene el lobo! \#i Viene el lobo!

(38) iQue si vienes mañana! *iSi vienes mañana!

(39) iQue vayas a comer! * *Vayas a comer!

(40) iQue digan eso de ti! *iDigan eso de ti!

El comportamiento ante la supresión está relacionado con los diferentes contextos de uso: cuando que funciona como partícula citativa (ejs. 38,39 y 40) el resultado es agramatical. En cambio, en los usos modales no citativos (tradicionalmente descritos como "sorpresa") que alterna con el subjuntivo y su ausencia es suplida por el modo verbal. La presencia de que inicial átono con indicativo, en (37), produce un enunciado no agramatical, pero sí ausente de una especificación modal; el resultado no es agramatical, pero tampoco totalmente correcto.

El uso de (30) entra de lleno en los usos citativos anteriormente citados, puesto que marca la proposición como atribuible a otra persona y resulta inadecuado establecer una identidad entre la fuente del mensaje y el transmisor de la misma; cuando esto ocurre, varía la interpretación del enunciado, que se considera, no ya una marca atributiva, sino de matización (es decir, su interpretación es idéntica a la de 31 abajo):

\section{(31') \#Que digo yo ${ }_{i}$ que te llamaré pronto}

En el ejemplo (31), que posee un valor atenuador, entendiendo por atenuador todo uso que haga referencia a una escala contrastiva de dos elementos, agrupados en torno al eje <atenuado, neutro> (Albelda, 2000). En (31), la supresión de que tiene como resultado producir un enunciado neutro (31. a), que puede ser atenuado ulteriormente con la adición de otros parentéticos $(31 . \mathrm{b}, 31 . \mathrm{c})$ o puede ser reforzada con la introducción de un intensificador (31. d).

(31) a. Podemos comer ya (grado neutro)

(31) b. Digo yo que pademos comer ya (enunciado atenuado)

(31) c. Que podemos comer ya (id.)

(31) d. Fijo que podemos comer ya (enunciado intensificado)

La poesía popular hace uso del que inicial átono: 
Se puede apreciar en este ejemplo un uso muy similar al de (26) y (27), con la única diferencia de que, en este caso, no es necesario hacer referencia a un contexto previo (contexto de insistencia). Que puede aparecer en posición inicial absoluta y es su sola aparición la que legitima la idea de énfasis, lo que se comprueba comparando (32') con la más neutra (32):

\section{(32') De noche lo mataron/ al caballero [...]}

El carácter marcado de (32) muestra que la diferente interpretación de ambos enunciados se sitúa en la partícula, por lo que esta puede inducir la creación de un contexto que no está presente en el momento de su emisión. Aunque parece que esta interpretación es típicamente procedimental, obsérvese, sin embargo, que en (36) la conjunción podía ser suprimida sin pérdida de sus instrucciones. Interpretamos este dato como una prueba de que la información modal no está codificada solo en el léxico, pero que algunos ítems léxicos, en ocasiones, la pueden codificar.

Por último, en (33') se ejemplifican los casos en que que sirve para el cambio de tópico:

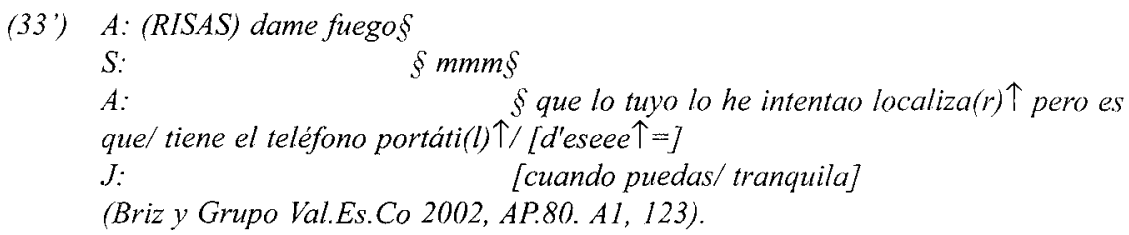

Una vez más, el enunciado puede prescindir de que sin afectar la comprensión del mensaje, pero produciendo un cambio en la escala <neutro, intensificado $>$. Este refuerzo del enunciado contribuye al cambio de tema.

Se puede ver cómo, en los datos que acabamos de reproducir, el que inicial átono, tanto en contextos interrogativos como afirmativos o exclamativos, ocupa una posición externa a la oración, ya que puede coocurrir con un que tónico (ejs. 11, 16, 17 y 19) o con otros pronombres (ej. 14) en las oraciones interrogativas y ocupa una posición extraproposicional en las afirmativas. La atonicidad y la posición inicial son rasgos que han llevado a una equiparación entre este que y el introductor de completivas, pero tales rasgos muestran una homogeneidad solo aparente. No hay que olvidar que la tendencia a ocupar la posición inicial es una característica general de los elementos que funcionan como marcadores discursivos. La marcación discursiva es un hiperónimo de varias funciones pragmáticas, entre las que se incluye y de entre las que sobresale la conexión, pero que no es en modo alguno equiparable. No solo los conectores muestran una marcada preferencia por la posición inicial. Marcadores de relaciones interactivas como oye o mira o elementos modales como bueno o claro muestran una misma preferencia posicional (Pons, 1998), lo que quiere decir que la primera posición de un enunciado no es una pista inequívoca de la existencia de conexión, sino que está marcada para la expresión de la marcación discursiva.

Hasta ahora hemos realizado una interpretación modal de los usos de que inicial átono. Esta interpretación presenta dos ventajas sobre la unitiva: en primer lugar, no necesita hacer 
referencia a elementos sobrentendidos, que plantean problemas de distinto tipo (vid. Sección 1). En segundo lugar, permite una interpretación unificada de todos los usos de que inicial átono, salvando el hiato que separaba, anteriormente, los valores en afirmativas y exclamativas, por un lado, de los valores interrogativos, por otro. Pero para que esta explicación esté articulada, es necesario responder aún a varias preguntas: ¿Qué tipo de categoría es la modalidad? ¿Cómo se estructura? ¿Pertenece la modalidad a la partícula o forma parte de la construcción?

Consideramos la conexión una categoría pragmática. Esto quiere decir que la equiparación existente entre sus miembros no se establece en función de identidades formales, sino funcionales. En este sentido, pertenecen a la categoría modalidad aquellos elementos cuyas ocurrencias presenten valores modales. En otras lenguas, como el alemán, la modalidad pertenece a la gramática, lo que significa que existe una clase cerrada de palabras (partículas modales), que se puede distinguir de clases vecinas (palabras modales) en función de una serie de criterios formales y funcionales y que ocupan un lugar fijo en la oración. En español, esta equiparación entre sintaxis y modalidad no se da, lo que quiere decir que su expresión es pragmática y que se manifiesta como una recarga secundaria de funciones en algunos elementos, entre los que se encuentran muchos conectores prototípicos (que, pues, pero, etc). Cuando decimos que la modalidad es una categoría pragmática estamos haciendo hincapié en esta identidad funcional.

Los valores modales se pueden agrupan en torno a una serie de rasgos: el binomio acuerdo/desacuerdo, típicamente interactivo, el binomio refuerzo/atenuación y el rasgo atribución de voz. Los dos primeros pares están íntimamente relacionados, ya que el acuerdo con algo contribuye a reforzarlo, y el desacuerdo muchas veces lleva aparejada la atenuación del enunciado (sobre todo en aquellos casos en los que coincide con una respuesta despreferida). En última instancia, estos cuatro valores se relacionan con el hablante, que es quien manifiesta su compromiso con el contenido transmitido en todos los casos. El rasgo atribución de voz marca la ausencia de compromiso del hablante ante lo dicho; al pertenecer a otro enunciador, los rasgos anteriores no actúan. Como se ha podido comprobar, todos los usos de que inicial átono caen en alguno de estos tres valores: los valores citativos de las interrogativas (19-23), los valores de refuerzo $(24-30,32)$ y los de atenuación (31).

Queda por dilucidar la cuestión de si la modalidad se inserta o no en el léxico. Los datos parecen indicar que la modalidad en ocasiones parece inducida a partir de la presencia de que, siendo en otras el resultado de una construcción a cuyo significado final contribuyen también la entonación exclamativa, el subjuntivo y la posición inicial. Los usos del lenguaje literario serían ejemplos del primer caso, mientras que ejemplos como (13) -construcción focal-, (24) -supuesto uso regional-o (27)-(29) -contextos de insistencia y deseos con subjuntivo optativo- parecen explicarse mejor como resultado de la coocurrencia de varios factores. Se añade a esto la dificultad de explicar cómo la información de modalidad archivada en una palabra se realizaría en cada caso y qué factores bloquearían su aparición (Pustejovski, 1995). En cualquier caso, esta cuestión queda abierta.

Vistas las relaciones entre conexión y modalidad, y la estructura interna de esta última, es necesario determinar el peso que cada uno de estos valores tiene en la descripción de que, y cómo se pueden conciliar los valores gramaticales, conectivos y modales de esta forma. 


\section{Un Esquema General para (los) usos de Que}

En Pons Bordería (1998), el análisis cuantitativo de más de tres mil ocurrencias de once elementos con características conectivas daba como resultado ${ }^{6}$ un dibujo de la conexión en español como una categoría radial. Alrededor de un centro categorial, formado por las conjunciones de coordinación más frecuentes, se situaban los demás elementos formando la siguiente estructura:

Entre el centro y la periferia de la categoría, formada esta por los apelativos oye, mira y las partículas modales bueno y claro, existe una zona intermedia donde se sitúan elementos de naturaleza mixta, como entonces y o sea. En este dibujo, como se puede comprobar, que forma parte de los conectores prototípicos.

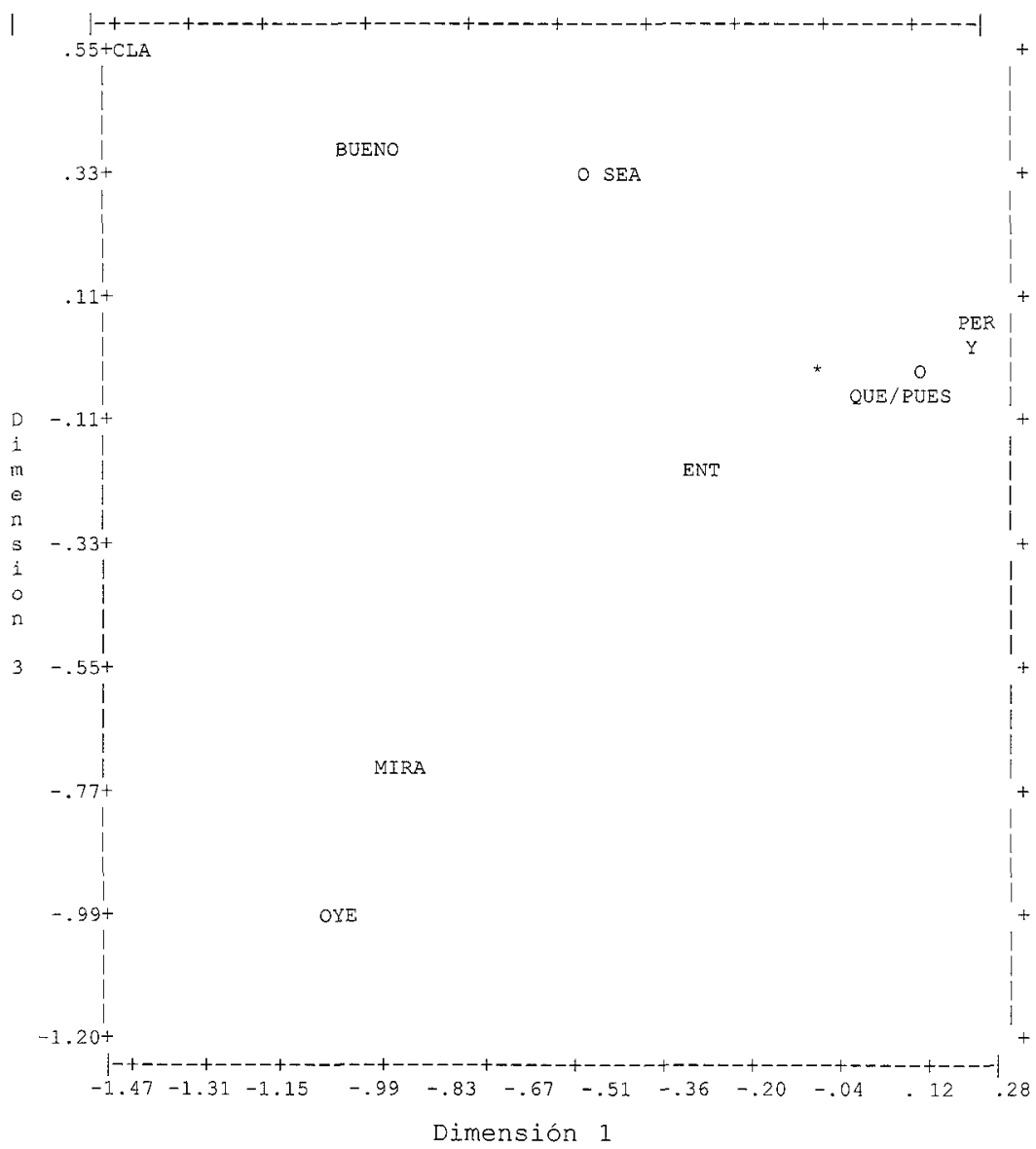

Figura 1: Resultados de un análisis de correspondencias sobre once conectores (Pons, 1998)

\footnotetext{
6 El análisis consistió en un estudio multivariante de las veinte preguntas distintas de cada una de las más de tres mil fichas. Los sesenta mil datos resultantes fueron agrupados siguiendo técnicas estadisticas de reducción dimensional. El resultado es un dibujo del prototipo de la categoria conexión obtenido por métodos estadísticos y, al menos en una fase del análisis, objetivos.
} 
Ahora bien, ¿cómo es posible compatibilizar esta caracterización de que como conector prototípico con los datos presentados en este trabajo? Para ello hay que tener en cuenta que la descripción de un elemento no termina con su inclusión dentro de una categoría, sino que debe incluir, además, la distribución de sus usos. Si la categoría se estructura en torno a un prototipo radial, los usos de una forma determinada adoptan la estructura de parecido de familia. La razón para esta diferencia es que, mientras que la categoría pragmática conexión se define en términos de semejanza funcional (es decir, son conectores las unidades que en mayor número de casos funcionen como tales) y, por tanto, a mayor cantidad de usos conectivos y a mayor cantidad de características propias de la conexión mayor su centralidad, los valores de una palabra, sin embargo, plantean una relación entre forma y funciones que viene determinada por la evolución de cada elemento en particular, por lo que es de esperar que no haya identidad funcional entre todos sus usos y que una misma forma pueda, por ello, funcionar en varias categorias de forma simultánea. Reducir la relación forma-función a una proyección uno-a-uno produce consecuencias indeseables, como tener que decidir entre asignar a $y$, por ejemplo, estatuto de conjunción o estatuto de conector, o diferenciar usos conectivos de no conectivos, como ocurre entre entonces adverbio y entonces conjunción. La relación que vincula formas y funciones para el caso de los conectores no es biyectiva, sino suprayectiva.

Es de esperar, desde esta perspectiva, que el estudio de los valores de un conector lleve a descubrir usos no conectivos, lo que no debe llevar a rechazarlos, ni a unificarlos en un mismo paradigma, sino a explicar la relación entre los ámbitos funcionales relacionados. En el caso de la conexión, hemos propuesto (Pons, 2000 y 2004) una relación entre las categorías conexión, modalidad y la relación interactiva que mantienen los participantes como categorías pragmáticas vecinas.

En el caso de que, como en el de otros conectores (pero o entonces), la dimensión modal adquiere un peso importante en el conjunto de sus valores no conectivos. Más que interpretar este dato en el sentido de distinguir varios tipos de que, preferimos situar la forma en la intersección de varias categorías pragmáticas, como la conexión y la modalidad. El carácter gradual de las categorías encaja bien con esta visión de sus usos, con lo que los dos tipos de prototipo empleados, el radial y el de parecido de familia, producen una descripción coherente de la clase y de sus formas.

Queda ahora por integrar las conclusiones de este artículo en una visión más general del funcionamiento de que. No vamos a proponer una visión de todos sus usos, porque tenemos en mente la observación -o advertencia- que hiciera Bello en su Gramática, al referirse a este elemento: "no hay palabra castellana que sufra tan variadas y a veces inexplicables transformaciones" (Bello, 1988, 1847: 624), pero sí que es posible reducir su aparentemente caótica diversidad funcional a un esquema parcial, tal y como se articula en el siguiente cuadro:
Que
Tónico
Átono

Pronombre

Interrogativo

Conector

Modalizador

Relativo

usos conjuntivos, que inespecífico

que inicial 
Teniendo en cuenta solo la forma y a partir de la distinción entre usos de qué tónico y qué átono, se pueden distinguir, por un lado, los valores como pronombre interrogativo y relativo de que; por otro, los valores conectivos, que comprenden, a su vez, los valores gramaticales (que introductor de completivas, como formante en construcciones consecutivas y comparativas, etc.) y los no gramaticales (los usos de que inespecífico, en los que las etiquetas gramaticales no se aplican). Finalmente, los valores modales, en los que la conjunción deja de ser tal y se convierte en marca de la actitud del hablante hacia lo dicho, que se subdivide, a su vez, en refuerzo/atenuación, acuerdo/desacuerdo y atribución de voz (es decir, compromiso del hablante frente a no compromiso).

Para acabar, una última precisión: el acercamiento no discreto a los usos de que exige tomar el esquema precedente como desde una visión prototípica, por lo que no se debe considerar que todos los usos de que inicial átono indiquen solo y únicamente valores modales (como se vio en 3.1, sirve también para introducir estructuras focales), sino que este es un uso preferente.

\section{Referencias bibliográficas}

Acosta, L. (1984): "Las partículas modales del alemán y español", Studia Philologica Salmanticensia, 7-8, págs. 7-41.

Albelda Marco, M. (2000): "La intensificación del decir: el caso de fijo", XXX Congreso de la SEL, Madrid.

Alcina Franch, J. y J. M. Blecua (1975): Gramática española. Barcelona, Ariel.

Beinhauer, W. (1978: 1929): El español coloquial. Madrid, Gredos.

Bello, A., et alli (1988: 1847): Gramática de la lengua castellana destinada al uso de los americanos. Madrid, Arco.

Berrendonner, A. (1983): "Connecteurs pragmatiques et anaphore". Connecteurs pragmatiques et structure du discourse. Actes du 2ème Colloque de Pragmatique de Génève. Génève, Cahiers de linguistique française, págs. 214-246.

Deulofeu, J. (1988): "Syntaxe de que en français parlé et le problème de la subordination", Recherches sur le français parlé, 8, págs. 79-104.

Dendale, P. and L. Tasmowski (2001). "Introduction: Evidentiality and related notions", Journal of Pragmatics 33 , págs. 339-348

Escandell Vidal, M. V. (1999). "Los enunciados interrogativos. Aspectos semánticos y pragmáticos".

En I. Bosque y V. Demonte (eds): Gramática descriptiva de la lengua española. Madrid, EspasaCalpe, págs. 3929-3991.

Ferrer Mora, H. (2002): "Die dt. Modalpartikeln und die sp. «conectores», oder: dt. Konnektoren und sp. Modalpartikeln? Stand der Forschung Deutsch-Spanisch". Actas del 34th Colloquium of Linguistics. Johannes Gutenberg-Universität, FASK Germersheim, Peter Lang, págs. 415-424.

Gadet, F., et Mazière. (1987): "L'extraordinaire souplesse du strument que", Le Français Moderne, 55, págs. 204-215.

Garrido Medina, J. (1998): "Discourse structure in grammar". Estudios ingleses de la Universidad Complutense 6, págs. 33-48.

Garrido Medina, J. (1999): "Los actos de habla". En I. Bosque y V. Demonte (eds): Gramática descriptiva de la lengua española. Madrid, Espasa-Calpe, cap. 60.

Gili Gaya, S. (1983): Curso superior de sintaxis española. Barcelona, Bibliograf.

Kany, C. E. (1969: 1945): Sintaxis hispanoamericana. Madrid, Gredos.

Lenz, R. (1920): La oración y sus partes. Madrid, Revista de Filología Española. 
Levinson, S. (1989): Pragmática. Barcelona, Teide.

Martín Zorraquino, M. A. (1991): "Spanisch: Partikelforschung (Partículas y modalidad)". En Holtus, G., M. Metzeltin y C. Schmitt (eds.): Lexicon der Romanistischen Linguistik. Tübingen, Max Niemeyer, págs. 111-125.

Pons Bordería, S. (1998). Conexión y conectores. Estudio de su relación en el registro informal de la lengua. Valencia, Cuadernos de Filología.

Pons Bordería, S. (2000): "Los conectores". A. Briz y Grupo Valesco (eds.): ¿Cómo se comenta un texto coloquial? Barcelona, Ariel, págs. 198-230.

Pons Bordería, S. (en prensa). "A Functional Approach for the Study of Discourse Markers". En K. Fischer (ed): Approaches to Discourse Particles. Amsterdam, Elsevier.

Porroche, M. (2000): "Algunos aspectos del uso de que en el español conversacional (que como introductor de oraciones 'independientes'", Círculo de Lingüistica Aplicada a la Comunicación 3: http: //www.ucm.es/info/circulo/no3/porroche.htm (septiembre de 2000).

Pustejovski (1995): The generative lexicon. Cambridge, The MIT Press.

Real Academia Española (1854): Gramática de la Lengua Castellana. Madrid, Imprenta nacional.

Salvá, V. (1988: 1835): Gramática de la lengua castellana. Madrid, Arco (ed. de Margarita Lliteras).

Schwenter, S. (1999): Pragmatics of conditional marking; implicature, scalarity and exclusivity. Nueva York, Garland.

Spitzer, L. (1942): "Notas sintáctico-estilísticas a propósito del español que", RFH 4, 2, págs. 105 126.

Súñer, A. (1999): "Las interrogativas indirectas". En I. Bosque y V. Demonte (eds): Gramática descriptiva de la lengua española. Madrid, Espasa-Calpe, págs. 2149-2195.

Vigara Tauste, A. M. (1992): Morfosintaxis del español coloquial. Madrid, Gredos. 